\title{
Feasibility of the AtriClip Pro Left Atrium Appendage Elimination Device via the Transverse Sinus in Minimally Invasive Mitral Valve Surgery
}

\author{
Tomonori Shirasaka, M.D., Ph.D., Shingo Kunioka, M.D., Masahiko Narita, M.D., Ryohei Ushioda, M.D., \\ Keisuke Shibagaki, M.D., Yuta Kikuchi, M.D., Naohiro Wakabayashi, M.D., Natsuya Ishikawa, M.D., \\ Hiroyuki Kamiya, M.D., Ph.D. \\ Department of Cardiac Surgery, Asahikawa Medical University, Asahikawa, Japan
}

\section{ARTICLE INFO}

Received May 26, 2021

Revised July 27, 2021

Accepted July 27, 2021

Corresponding author

Shingo Kunioka

Tel 81-166-68-2494

Fax 81-7035134247

E-mail skunioka44@gmail.com;

kyokui090069@gmail.com

ORCID

https://orcid.org/0000-0002-7451-5264

\begin{abstract}
Background: Achieving external access to and manual occlusion of the left atrial appendage (LAA) during minimally invasive mitral valve surgery (MIMVS) through a small right thoracotomy is difficult. Occlusion of the LAA using an epicardial closure device seems quite useful compared to other surgical techniques.

Methods: Fourteen patients with atrial fibrillation underwent MIMVS with concomitant surgical occlusion of the LAA using double-layered endocardial closure stitches ( $n=6$, endocardial suture group) or the AtriClip Pro closure device ( $n=8$, Atriclip group) at our institution. The primary safety endpoint was any device-related adverse event, and the primary efficacy endpoint was successful complete occlusion of blood flow into the LAA as assessed by transthoracic echocardiography at hospital discharge. The primary efficacy endpoint for stroke reduction was the occurrence of ischemic or hemorrhagic neurologic events.

Results: All patients underwent LAA occlusion as scheduled. The cardiopulmonary bypass and aortic cross-clamp times in the endocardial suture group and the Atriclip group were $202 \pm 39$ and $128 \pm 41$ minutes, and $213 \pm 53$ and $136 \pm 44$ minutes, respectively ( $p=0.68$, $\mathrm{p}=0.73)$. No patients in either group experienced any device-related serious adverse events, incomplete LAA occlusion, early postoperative stroke, or neurologic complication. Conclusion: Epicardial LAA occlusion using the AtriClip Pro during MIMVS in patients with mitral valve disease and atrial fibrillation is a simple, safe, and effective adjunctive procedure.
\end{abstract}

Keywords: AtriClip Pro, Minimally invasive cardiac surgery, Left atrium appendage

\section{Introduction}

Surgical occlusion of the left atrial appendage (LAA) is important for stroke prevention in patients with mitral valve disease and atrial fibrillation (AF) [1]. Various surgical or interventional methods have been reported for LAA occlusion [2-4]. However, conventional procedures, such as external ligation or the use of purse-string or internal running sutures, are not always promising or reproducible and occasionally fail to achieve complete LAA occlusion $[2,3]$. An epicardial LAA closure device seems feasible due to its technical simplicity and time-saving nature [4-6].
Caliskan et al. [6] recently reported the safety and durability of the AtriClip device (AtriCure, Mason, OH, USA) for LAA occlusion in conventional open-heart surgery in their first-in-humans prospective human trial, and they emphasized the potential efficacy of this adjunctive procedure for stroke prevention. However, during minimally-invasive mitral valve surgery (MIMVS) through a small right thoracotomy, the surgical workspace is very limited and the distance from the right chest wall to the LAA is very long, making it quite difficult to grasp the LAA manually. Therefore, a closure device with a shaft long enough to reach the LAA seems quite reasonable as a way to access 
the LAA safely without injury, which is devastating during minimally invasive cardiac surgery (MICS) mitral surgery.

We performed MICS mitral valve surgery with concomitant LAA occlusion using the upgraded AtriClip Pro device in several patients with mitral valve disease and a clear history of persistent or paroxysmal AF. Therefore, we reviewed the clinical feasibility of the improved version of the device during MICS mitral valve surgery.

\section{Methods}

The institutional review board of Asahikawa Medical University Hospital approved this retrospective study (IRB approval no., 19083) and waived the need for written patient consent.

\section{Patients' demographics and the change in the maneuver for left atrial appendage elimination}

Our indications for LAA exclusion in MIMVS are (1) chronic or paroxysmal AF history, (2) history of cerebral infarction, and (3) a CHADS2 score $\geq 2$. Since the commencement of MIMVS at Asaikawa Medical University, 12 patients with significant mitral valve stenosis or insufficiency with a clear history of persistent or paroxysmal AF and 2 patients with a history of cerebral infarction underwent LAA exclusion in MIMVS. Before introducing AtriClip Pro, 6 patients underwent LAA exclusion with endocardial sutures ( $n=6$, endocardial suture group).

Since the introduction of AtriClip Pro, 8 patients $(n=8$, AtriClip group) underwent MICS mitral valve surgery with concomitant surgical occlusion of the LAA using the AtriClip Pro epicardial closure device, and the characteristics of patients in these two groups were reviewed (Table 1).

\section{Operative procedure of MICS mitral valve surgery}

A 5- to 7-cm right thoracotomy was performed via the fourth intercostal space. Femoral cannulation was performed with a 1-stage arterial cannula (PCKC-A, 16F or 18F; Toyobo, Osaka, Japan) and a 2-stage venous cannula (RAP FV cannula, 22F or 25F LivaNova, Milan, Italy) placed through the right atrium into the superior vena cava. After aortic cross-clamping with a Cygnet flexible clamp (Vitaltec International Inc., Plymouth, MA, USA), initial cardioplegia was delivered in an antegrade fashion, followed by left atriotomy with intermittent delivery of cardioplegia performed in a retrograde fashion via the coronary sinus.
Ablation was performed as follows: after retracting the left atrial wall upward and exposing the mitral valve, energy was applied using cryoablation probes (CryoICE, incision; AtriCure) 3 times to complete a box-shaped lesion around the pulmonary veins connected to the edge of the left atriotomy incision.

\section{Delivery of the AtriClip Pro}

The AtriClip Pro device is a clip consisting of 2 parallel rigid titanium tubes with elastic nitinol springs covered by a knit-braided polyester sheath. Before commencing the operation, the LAA was assessed by transesophageal echocardiography (TEE) to rule out the presence of an intra-arterial thrombus. The base of the LAA was measured. Four different-sized clips are available $(35,40,45$, and $50 \mathrm{~mm}$ ) according to the LAA base measurement.

We routinely deployed AtriClip Pro before mitral valve surgery to reconfirm whether LAA exclusion was appropriately completed from the inside of the left atrium. The LAA was exposed by gentle retraction of the ascending aorta using a left atrial retractor (Adams-Yozu Mini-Valve System; Geister, Tuttlingen, Germany) via the transverse sinus. The clip was placed at the LAA base, avoiding the circumflex and pulmonary arteries. The first step to confirm the distal edge of LAA is to pick the LAA up gently with forceps and to confirm the base of LAA. The second step is to do a shoulder check of the shape of LAA and the length of the LAA base with a flexible sizer referred to as a selection guide. We do not use a retracting stitch onto the LAA to avoid tearing the LAA unexpectedly. In the case of inappropriate placement, the clip could be repositioned before final deployment. Once the clip was positioned optimally, it was manually closed and released completely from the deployment device (Supplementary Video 1). Successful occlusion was defined as the absence of blood flow into the LAA when coming off cardiopulmonary bypass, as confirmed both by direct visual assessment and TEE after unclamping followed by resumption of the heartbeat (Fig. 1).

\section{Postoperative anticoagulation}

Postoperative oral anticoagulation was performed. Irrespective of the rhythm pattern, warfarin was administered routinely to all patients and the prothrombin time-international normalized ratio was controlled between 1.8 and 3.0. If the sinus rhythm was stable for longer than 3 months, warfarin therapy was discontinued except in patients who underwent mitral valve replacement with a mechanical valve. 
Table 1. Baseline and demographic variables of patients undergoing MICS mitral valve surgery in the AtriClip and the endocardial suture groups

\begin{tabular}{|c|c|c|c|}
\hline Characteristic & AtriClip $(n=8)$ & Endocardial suture $(\mathrm{n}=6)$ & p-value \\
\hline Age $(y r)$ & $73 \pm 12$ & $66 \pm 14$ & 0.31 \\
\hline Sex (male) & 4 & 4 & 0.56 \\
\hline NYHA class $\geq$ III & 2 & 0 & 0.42 \\
\hline CHADS 2 score & $2.5 \pm 0.8$ & $1.7 \pm 1.0$ & 0.16 \\
\hline CHA2DS2-VASc score & $3.8 \pm 1.2$ & $1.8 \pm 1.3$ & $0.02^{*}$ \\
\hline Antiarrhythmic medication & 1 & 1 & - \\
\hline \multicolumn{4}{|l|}{ Cardiac and related medication } \\
\hline ACE-I or ARB & 3 & 2 & 0.56 \\
\hline Anticoagulants/antiplatelet & 5 & 3 & 0.81 \\
\hline Beta blockers & 2 & 2 & - \\
\hline Calcium channel blockers & 2 & 1 & 0.51 \\
\hline Diuretics & 5 & 4 & 0.87 \\
\hline \multicolumn{4}{|l|}{ Preoperative TTE } \\
\hline LVDd (mm) & $48 \pm 7.6$ & $53 \pm 8.9$ & 0.33 \\
\hline LVDs (mm) & $32 \pm 6.4$ & $36 \pm 7.0$ & 0.33 \\
\hline $\mathrm{EF}(\%)$ & $61 \pm 7.9$ & $60 \pm 10.9$ & 0.86 \\
\hline LA size $(\mathrm{mm})$ & $60 \pm 10.6$ & $54 \pm 8.6$ & 0.29 \\
\hline $\mathrm{LAV}(\mathrm{mL})$ & $147 \pm 60.7$ & $140 \pm 49.7$ & 0.89 \\
\hline $\mathrm{LAVI}\left(\mathrm{mL} / \mathrm{m}^{2}\right)$ & $93.2 \pm 37.4$ & $85.8 \pm 32.0$ & 0.72 \\
\hline LA thrombus (\%) & 0 & 0 & - \\
\hline \multicolumn{4}{|l|}{ Preoperative computed tomography } \\
\hline Vertebra-sternum distance (mm) & $108 \pm 13$ & $105 \pm 19$ & 0.78 \\
\hline \multicolumn{4}{|l|}{ Criteria for inclusion } \\
\hline History of AF & 5 & 6 & 0.76 \\
\hline CHADS score $>2$ & 4 & 1 & 0.24 \\
\hline Age $>75 \mathrm{yr}$ & 3 & 3 & 0.92 \\
\hline Hypertension and age $>65 \mathrm{yr}$ & 4 & 1 & 0.24 \\
\hline Previous stroke & 0 & 0 & - \\
\hline
\end{tabular}

Values are presented as mean \pm standard deviation or number.

MICS, minimally invasive cardiac surgery; NYHA, New York Heart Association; ACE-I, angiotensin converting enzyme inhibitor; ARB, angiotensin II receptor blocker; TTE, transthoracic echocardiography; LVDd, left ventricular diastolic dimension; LVDs, left ventricular systolic dimension; EF, ejection fraction; LA, left atrium; LAV, left atrium volume; LAVI, left atrium volume index; AF, atrial fibrillation. p $<0.05$.

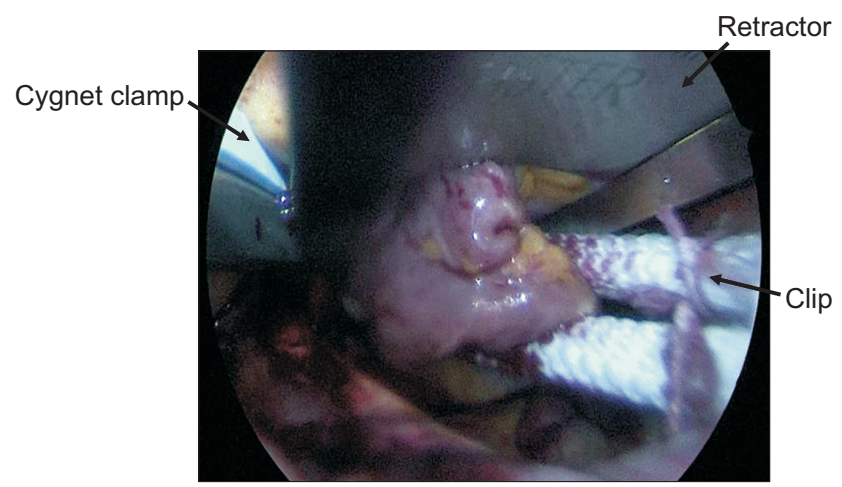

Fig. 1. Left atrial appendage (LAA) occlusion by clipping the base of the LAA with the AtriClip Pro via the transverse sinus.

\section{Endpoint}

The primary safety endpoint was any perioperative device-related adverse event. The primary efficacy endpoint for successful LAA occlusion was complete occlusion of blood flow into the LAA as assessed by postoperative transthoracic echocardiography (TTE) at hospital discharge. The primary efficacy endpoint for stroke reduction was freedom from ischemic or hemorrhagic neurologic events.

\section{Statistical analysis}

Preoperative, intraoperative, and postoperative data were recorded and reviewed retrospectively. Continuous variables were expressed as mean values \pm standard deviation, 
and categorical variables were presented as either absolute numbers or percentages.

\section{Results}

The overall clinical outcomes are shown in Tables 2 and 3. All patients underwent LAA occlusion as scheduled. There were no intraoperative procedure-related or device-related perioperative complications, such as damage to the left circumflex and pulmonary arteries, bleeding from an LAA base injury, or migration of the device after placement.

Table 2. Concomitant cardiac operations and efficacy of LAA occlusion by direct visual assessment and TEE intraoperatively in the AtriClip and the endocardial suture groups

\begin{tabular}{lccc}
\hline \multicolumn{1}{c}{ Surgical procedure } & $\begin{array}{c}\text { AtriClip } \\
(\mathrm{n}=8)\end{array}$ & $\begin{array}{c}\text { Endocardial } \\
\text { suture }(\mathrm{n}=6)\end{array}$ & p-value \\
\hline Mitral valve & 8 & 6 & - \\
$\quad$ Repair & 5 & 4 & 0.56 \\
$\quad$ Replacement & 3 & 2 & 0.56 \\
Tricuspid valve repair & 8 & 5 & 0.30 \\
Surgical maze procedure & 8 & 6 & - \\
Size of AtriClip Pro (mm) & $45 \pm 5$ & - & - \\
Success of LAA exclusion & 8 & 6 & - \\
By visual assessment & 8 & 6 & - \\
By TEE & 8 & 6 & - \\
\hline
\end{tabular}

Values are presented as number or mean \pm standard deviation.

LAA, left atrium appendage; TEE, transesophageal echocardiography.

Table 3. Operative time, CPB time, aortic cross-clamp time, and postoperative adverse events after left atrium appendage occlusion in the AtriClip and the endocardial suture groups

\begin{tabular}{lccc}
\hline \multicolumn{1}{c}{ Variable } & $\begin{array}{c}\text { AtriClip } \\
(\mathrm{n}=8)\end{array}$ & $\begin{array}{c}\text { Endocardial } \\
\text { suture }(\mathrm{n}=6)\end{array}$ & p-value \\
\hline Operative time (min) & $276 \pm 79$ & $236 \pm 23$ & 0.26 \\
CPB time (min) & $213 \pm 53$ & $202 \pm 39$ & 0.68 \\
Aortic cross-clamp time (min) & $136 \pm 44$ & $128 \pm 41$ & 0.73 \\
Adverse event & & & \\
Device-related event & 0 & - & - \\
Clip malposition & 0 & - & - \\
Postprocedural hemorrhage & 0 & 0 & - \\
Postoperative AF (at discharge) & 2 & 1 & 0.51 \\
Atrial-ventricular block & 0 & 0 & - \\
Congestive heart failure & 0 & 0 & - \\
Surgical site infection & 0 & 0 & - \\
Renal failure & 0 & 0 & - \\
Aortic dissection & 0 & 0 & - \\
Pulmonary embolism & 0 & 0 & - \\
\hline
\end{tabular}

Values are presented as mean \pm standard deviation or number. $\mathrm{CPB}$, cardiopulmonary bypass; $\mathrm{AF}$, atrial fibrillation.
LAA occlusion was successful in all patients, as assessed visually by the surgeon and by intraoperative TEE. Intraoperative TEE and postoperative TTE at hospital discharge confirmed the success of the procedures and LAA closure without residual perfusion.

No patients in either group had any clinical complications or neurologic disturbances after hospital discharge, and none had any postoperative device-related serious adverse events, such as postoperative stroke (Table 3).

\section{Discussion}

Based on the results of our study on the efficacy of the AtriClip Pro device in MIMVS via small right thoracotomy, we believe that this device is useful for MIMVS. The AtriClip Pro device is a new version based on the original AtriClip device (AtriCure Inc.). The difference from the previous version is that the top joints attached to the LAA clip can be articulated manually to aid in the proper placement of the clip (Fig. 2).

The EXCLUDE trial previously demonstrated the safety and efficacy of the AtriClip device [5]. In this multicenter study, 71 patients underwent open cardiac surgery via full median sternotomy with LAA clipping using the AtriClip. The intraprocedural success rate was $95.7 \%$, without any device-related adverse events. Moreover, of 61 patients who underwent postoperative computed tomographic (CT) angiography, 98.4\% had successful occlusion of the LAA [5]. Caliskan et al. [6] reported long-term results of the AtriClip in a prospective human trial with $36 \pm 23$ months of follow-up. They found no device-related complications, and a subgroup analysis of patients with discontinued oral anticoagulation showed a relative risk reduction of $87.5 \%$ and an observed ischemic stroke rate of 0.5 per 100 patient-years [6].

The LAA is a primary source of embolic events in patients with AF [1]. Therefore, LAA occlusion is vital to de-

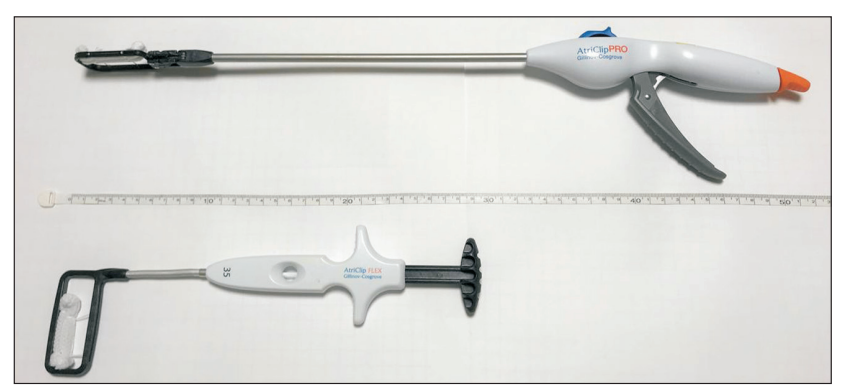

Fig. 2. Comparison of the rigid shaft length between the AtriClip Pro and the AtriClip (old version). 
crease the incidence of stroke. The most recent 2016 European Society of Cardiology guidelines for the management of AF states that interventional LAA occlusion may be considered in patients with high stroke risk and contraindications for long-term oral anticoagulation (class IIb) [7]. Similarly, the 2014 American Heart Association/American College of Cardiology/Heart Rhythm Society guidelines for the management of AF also advocate for the validity of LAA excision or occlusion in patients undergoing openheart surgery (class IIb) [8]. The typical surgical methods for isolating blood flow into the LAA are external cut-andsew closure, surgical ligation using a double row of running sutures sewing from within the left atrium, and stapling occlusion with or without excision. However, several studies have demonstrated that these methods have high rates (up to $33 \%$ to $55 \%$ ) of incomplete occlusion from blood flow $[3,9]$.

During the last 6 years, 134 patients underwent mitral valve surgery via median sternotomy at our institution. Of these patients, 41 patients underwent LAA exclusion with (7 cases) or without LAA closure devices (34 cases). Two patients who underwent external cut-and-sew of the LAA required re-exploration due to postoperative bleeding from the LAA suture line, and in 1 case, the control of bleeding from the posterior wall of the left ventricle was quite difficult after an endocardial suture of the LAA orifice, resulting in a significantly prolonged operative time. In contrast, no patients in the LAA closure device group experienced unexpected bleeding, which may imply the safety and feasibility of the LAA exclusion device. Therefore, we regarded the maneuver of LAA exclusion with the external cutand-sew method or endocardial sutures to be riskier than we had expected. Moreover, once surgical bleeding at the LAA or LA wall develops, it is quite difficult to control the bleeding from the suture line in the MICS approach. Therefore, before introducing the AtriClip Pro device, we intentionally limited the indications for LAA exclusion in patients with mitral valve disease and chronic AF.

A recent report on the outcomes of MIMVS in Japan based on a nationwide surgical database showed that concomitant antiarrhythmic surgery was performed in only $7 \%$ of cases, while the preoperative AF rate was $16 \%$ [10]. This phenomenon might be linked to the fact that the MICS approach for complicated cases is associated with a significant prolongation of the operative time [11]. Chronic mitral valve disease has a relatively high risk of significant tricuspid valve insufficiency and emergence of AF, and many cases ideally require a double valve technique with concomitant antiarrhythmic surgery and LAA occlusion.
However, in this situation, the aortic cross-clamp time is significantly longer than that during single mitral valve surgery. In this respect, the operative strategy and time management are substantive issues.

The AtriClip Pro device is simple and easy to use, and it has the advantage of being time-saving in these situations. Rapid deployment makes the operative time much shorter. However, in this study, there were no significant differences in the cardiopulmonary bypass and aortic cross-clamp times between the 2 groups despite the fact that 3 cases in the AtriClip group (50\%) were operated by a MICS trainee (T.S.), while all cases in the endocardial suture group were operated by a single veteran MICS surgeon (H.K.).

The knit-braided polyester sheath covering the clip is a soft material, which can reduce the risk of traumatic injury to the LAA. In addition, the rigid shaft length is $25 \mathrm{~cm}$ (Fig. 2), which allows the surgeon to access the LAA from the fourth or fifth right intercostal space. In addition, the availability of 4 different-sized clips enables easy complete clipping irrespective of the LAA shape and size.

Our study demonstrated acceptable cardiopulmonary bypass and aortic cross-clamp times during MIMVS with the LAA occlusion device without any device-related serious adverse events, incomplete LAA occlusion, or early postoperative stroke. To our knowledge, this is the first report on LAA occlusion via the transverse sinus with the AtriClip Pro device during MICS mitral valve surgery in Japan. This study suggests the potential efficiency and efficacy of epicardial LAA occlusion using this device for reducing the incidence of postoperative stroke. The AtriClip Pro device might be a useful tool for routine LAA occlusion in patients with a history of AF who undergo MICS mitral valve surgery.

\section{Limitation}

This study has some limitations. First, this is a preliminary report showing the safety and feasibility of AtriClip Pro in MIMVS, and its sample size is quite small. In Japan, this device has not entered into widespread use, possibly because of the unavailability of insurance reimbursement. Moreover, our follow-up periods are still short. However, the concept of AtriClip Pro is fundamentally the same as that of the old version, AtriClip Flex, which has been recognized as a reliable device for elimination of LAA, as reported previously [6]. It also seems important to recognize the strong point of the AtriClip Pro, which is that it can be used to access the LAA safely without injury in the limited working space. In terms of its safety and cost-effectiveness, 
this device has the potential to be more widespread in the near future. Second, this is a single-center experience with an observational design, and all the established disadvantages of this design apply. Third, the confounding influence of concomitant surgical ablation on freedom from postoperative stroke could not be eliminated. Fourth, the gold-standard imaging technique for confirming LAA occlusion is CT angiography. In this study, 7 patients in the AtriClip group (87.5\%) were elderly (over 70 years old) and most of them had a history of chronic kidney disease. We intentionally did not perform CT angiography in those patients due to concerns about the preservation of renal function. However, we have changed our policy and now routinely perform $\mathrm{CT}$ angiography to confirm the elimination of LAA in patients who are relatively young and have normal kidney function.

\section{Conclusion}

LAA occlusion via the transverse sinus with the AtriClip Pro device during MIMVS is quite useful and effective at eliminating blood inflow into the LAA.

\section{Conflict of interest}

No potential conflict of interest relevant to this article was reported.

\section{ORCID}

Tomonori Shirasaka: https://orcid.org/0000-0001-9429-7025 Shingo Kunioka: https://orcid.org/0000-0002-7451-5264 Masahiko Narita: https://orcid.org/0000-0001-8150-2552 Ryohei Ushioda: https://orcid.org/0000-0001-6690-5759 Keisuke Shibagaki: https://orcid.org/0000-0001-9677-0058 Yuta Kikuchi: https://orcid.org/0000-0003-1361-6456 Naohiro Wakabayashi: https://orcid.org/0000-0002-0918-8623 Natsuya Ishikawa: https://orcid.org/0000-0002-8845-6488 Hiroyuki Kamiya: https://orcid.org/0000-0001-5623-1279

\section{Supplementary materials}

Supplementary materials can be found via https://doi. org/10.5090/jcs.21.048. Supplementary Video 1. The video of LAA exclusion with AtriClip Pro.

\section{References}

1. Blackshear JL, Odell JA. Appendage obliteration to reduce stroke in cardiac surgical patients with atrial fibrillation. Ann Thorac Surg 1996;61:755-9.

2. Kanderian AS, Gillinov AM, Pettersson GB, Blackstone E, Klein AL. Success of surgical left atrial appendage closure: assessment by transesophageal echocardiography. J Am Coll Cardiol 2008;52:9249

3. Lee R, Vassallo P, Kruse J, et al. A randomized, prospective pilot comparison of 3 atrial appendage elimination techniques: internal ligation, stapled excision, and surgical excision. J Thorac Cardiovasc Surg 2016;152:1075-80.

4. Park JH, Sohn SH, Choi JW, Park EA, Hwang HY. Safety and efficacy of left atrial appendage excision using a vascular stapler. Korean J Thorac Cardiovasc Surg 2020;53:127-31.

5. Ailawadi G, Gerdisch MW, Harvey RL, et al. Exclusion of the left atrial appendage with a novel device: early results of a multicenter trial. J Thorac Cardiovasc Surg 2011;142:1002-9.

6. Caliskan E, Sahin A, Yilmaz M, et al. Epicardial left atrial appendage AtriClip occlusion reduces the incidence of stroke in patients with atrial fibrillation undergoing cardiac surgery. Europace 2018;20: e105-14.

7. Kirchhof P, Benussi S, Kotecha D, et al. 2016 ESC guidelines for the management of atrial fibrillation developed in collaboration with EACTS. Europace 2016;18:1609-78.

8. January CT, Wann LS, Alpert JS, et al. 2014 AHA/ACC/HRS guideline for the management of patients with atrial fibrillation: a report of the American College of Cardiology/American Heart Association Task Force on practice guidelines and the Heart Rhythm Society. Circulation 2014;130:e199-267.

9. Katz ES, Tsiamtsiouris T, Applebaum RM, Schwartzbard A, Tunick PA, Kronzon I. Surgical left atrial appendage ligation is frequently incomplete: a transesophageal echocardiograhic study. J Am Coll Cardiol 2000;36:468-71.

10. Nishi H, Miyata H, Motomura N, et al. Which patients are candidates for minimally invasive mitral valve surgery?: establishment of risk calculators using National Clinical Database. Circ J 2019;83:167481 .

11. Falk V, Cheng DC, Martin J, et al. Minimally invasive versus open mitral valve surgery: a consensus statement of the international society of minimally invasive coronary surgery (ISMICS) 2010. Innovations (Phila) 2011;6:66-76. 